\title{
Películas de SRO-HFCVD como dieléctrico en estructuras MIS y sus propiedades eléctricas
}

\section{SRO-HFCVD films as dielectric in MIS structures and their electrical properties}

\author{
MARTÍNEZ-HERNÁNDEZ, Haydee Patricia†, LUNA-LÓPEZ, José Alberto, HERNÁNDEZ-DE LA \\ LUZ, José Álvaro David y CASCO-VASQUEZ, José Federico’’
}

Instituto Tecnológico de Apizaco, Departamento de Ingeniería Eléctrica y Electrónica, Tecnológico Nacional de México/Carretera Apizaco-Tzompantepec, esquina con Av. Instituto Tecnológico S/N. C.P. 90300. México.

'Benemérita Universidad Autónoma de Puebla, Av. San Claudio y 14 sur, Edificios IC5 y IC6, C.U., Col. San Manuel, Puebla, Pue. 72570, México.

ID $1^{\mathrm{er}}$ Autor: Haydee Patricia, Martínez-Hernández / ORC ID: 0000-0001-8863-4689, CVU CONACYT ID: 353253

ID $1^{\mathrm{er}}$ Coautor: José Alberto, Luna-López / ORC ID: 0000-0002-7647-3184, CVU CONACYT ID: 200808

ID $2^{\text {do }}$ Coautor: José Álvaro David, Hernández-De la luz / ORC ID: 0000-0002-7913-0240, CVU CONACYT ID: 240901

ID $3^{\text {er }}$ Coautor: José Federico, Casco-Vásquez, ORC ID: 0000-0002-9593-9760, CVU CONACYT ID: 74965

DOI: $10.35429 / J E E .2020 .12 .4 .10 .15$

Recibido Enero 15, 2020; Aceptado Junio 30, 2020

\section{Resumen}

Este trabajo presenta los resultados de la caracterización electro-óptica de una estructura MIS obtenida al depositar una película de un Óxido de Estaño dopado con Indio (ITO) sobre una película de Óxido de Silicio Rico en Silicio (SRO) usando el sistema de depósito químico en fase vapor activado por Filamento Caliente (HFCVD). Las películas SRO-HFCVD fueron depositadas considerando dos flujos de hidrógeno de 25 y $100 \mathrm{sccm}$, para crecer películas sencillas y dobles (bicapas) siendo ambas tratadas térmicamente a $1100^{\circ} \mathrm{C}$ para mejorar sus características ópticas y estructurales. Con las técnicas de Espectroscopia nula, XPS y fotoluminiscencia se hace el estudio que determina los espesores de las películas SRO, cuantifica el exceso de silicio presente en ellas y analiza sus espectros correspondientes. Estas películas funcionan como la capa activa en estructuras del tipo Metal-AislanteSemiconductor (MIS), y fueron caracterizadas eléctricamente a través de las curvas I-V. Del resultado de estas caracterizaciones se hace una comparación entre las estructuras MIS con películas vírgenes (V) y con las películas tratadas térmicamente (T-T). Los resultados obtenidos indican que las películas SRO-HFCVD con T-T modifican significativamente las propiedades ópticas y eléctricas de las estructuras MIS, lo que es prometedor para el diseño de sensores ópticos integrados.

\begin{abstract}
This paper presents the results obtained by the electrooptical characterization of a MIS structure built by depositing a film of an Indium Tin Oxide (ITO) on a Silicon Rich Oxide (SRO) film using the Chemical Vapor Deposition Hot Filament (HFCVD) system. The SROHFCVD films were deposited considering two hydrogen fluxes levels at 25 and $100 \mathrm{sccm}$, under these conditions we grow single and double films, both being heat treated at $1100{ }^{\circ} \mathrm{C}$ to improve their optical and structural characteristics. Through of the techniques of Null Spectroscopy, XPS and Photoluminescence, it was possible to determine the thickness of the SRO films, quantify the silicon excess present in them and analyze their spectra. These films are used as the active layer in Metal-Insulating-Semiconductor (MIS) structures, such structures were electrically characterized through the I-V curves. From the result of these characterizations a comparison is made between the MIS structures with films virgin (V) and with ones heat-treated (T-T). Characterizations indicate that SRO-HFCVD films with $\mathrm{T}-\mathrm{T}$ significantly modify the optical and electrical properties of MIS structures, which is promising for the design of integrated optical sensors.
\end{abstract}

Photoluminescence, XPS, MIS structures, I-V Curves

Fotoluminiscencia, XPS, Estructuras MIS, Curvas I-V

Citación: MARTÍNEZ-HERNÁNDEZ, Haydee Patricia, LUNA-LÓPEZ, José Alberto, HERNÁNDEZ-DE LA LUZ, José Álvaro David y CASCO-VASQUEZ, José Federico. Películas de SRO-HFCVD como dieléctrico en estructuras MIS y sus propiedades eléctricas. Revista de Ingeniería Eléctrica. 2020. 4-12:10-15.

\footnotetext{
*Correspondencia al Autor (Correo Electrónico: haydee.mh@ apizaco.tecnm.mx)

$\dagger$ Investigador contribuyendo como primer autor.
} 


\section{Introducción}

El uso de películas y dispositivos procesados a base de silicio son una ventaja clave, debido a su fabricación de alto rendimiento y bajo costo [13]. Así, aquellas estructuras que absorben y emiten luz a partir del silicio resultan ser de gran importancia en el campo de la electro-fotónica. En particular, uno de estos materiales es el óxido de silicio rico en silicio (SRO) el cual se considera como un material multifase constituido por una mezcla de sílice $\left(\mathrm{SiO}_{2}\right)$, óxidos no estequiométricos $(\mathrm{SiO} x, \mathrm{x}<2)$ y silicio elemental. Este material ha evolucionado a partir de que DiMaria et al. [4] observó electroluminiscencia en SRO por primera vez, así mismo Leight Caham [5] mediante un grabado electroquímico obtuvo emisión visible de silicio poroso.

El SRO ha sido fabricado por diferentes sistemas, siendo los más comunes los que usan la Deposición de Vapor Químico, como el LPCVD (Deposición de Vapor Químico a Baja Presión) [6, 8-10], el HFCVD (Deposición de Vapor Químico de Filamento Caliente) [6], y el PECVD (Deposición de Vapor Químico mejorada por plasma) [6-8], además de aquellos que usan el sol-gel [10], implante de silicio en $\mathrm{SiO}_{2}$ [11], y sputtering [6]. Todos estos sistemas son compatibles con la tecnología de fabricación de circuitos integrados y han dado lugar a novedosos dispositivos tales como guías de onda, memorias no volátiles, supresores de picos, detección de luz y dispositivos de emisión.

Las estructuras MIS para su fabricación, utilizan las películas SRO como la capa activa depositada sobre el sustrato de silicio y en la parte posterior de éste se deposita algún metal como Oro (Au) o Aluminio (Al) que se utiliza como contacto posterior. Mientras que sobre la capa activa se deposita una película de óxido de estaño dopado con indio (ITO), como óxido conductor transparente (TCO) en lugar de compuertas metálicas $[12,20]$.

Para la realización de este trabajo, las estructuras MIS están conformadas por películas SRO-HFCVD depositadas sobre silicio con contactos metálicos de Au y de ITO las cuales son energizadas eléctricamente, con el objetivo de analizar sus curvas I-V.
Dos flujos de hidrógeno molecular $\left(\mathrm{H}_{2}\right)$ de 25 y $100 \mathrm{sccm}$ fueron utilizados para la obtención de películas sencillas $\mathrm{SRO}_{25}$ y $\mathrm{SRO}_{100}$ y la combinación de estos flujos para las de doble película $\mathrm{SRO}_{25 / 100}$ y $\mathrm{SRO}_{100 / 25}$. Cabe señalar que las estructuras MIS de película sencilla y doble del tipo (V), presentan limitaciones de transporte para un alto voltaje de encendido el cual está cerca del voltaje de ruptura dieléctrica y brincos de corriente, después de los cuales se rompe el dieléctrico. Como una alternativa para mejorar las características ópticas, morfológicas y eléctricas en la estructura MIS, se aplicó el tratamiento térmico [12]. Observando en las estructuras con T-T que después del primer rompimiento la corriente aumenta lentamente con el voltaje, es decir, sigue controlando la corriente. También es notorio que originalmente el mínimo de la corriente a bajos voltajes esta desplazada de cero volts, probablemente debido al atrapamiento de carga en el SRO.

\section{Objetivo}

Correlacionar las caracterizaciones ópticas de las películas SRO-HFCVD sencillas y dobles con las curvas I-V de las estructuras MIS conformadas con estas películas sin y con tratamiento térmico.

\section{Desarrollo y metodología}

Las películas SRO fueron depositadas por el sistema HFCVD (Deposito Químico en fase Vapor activado por Filamento Caliente), donde se usaron sustratos de silicio, tipo P (100), de 2" de diámetro, con una resistividad de $1-5 \Omega$ y 300 micras de espesor, estos sustratos se limpiaron previamente mediante el proceso estándar MOS [12]. El sistema HFCVD, utiliza flujo de hidrógeno molecular el cual se disocia a $2000^{\circ} \mathrm{C}$, esta temperatura se alcanza con 11 filamentos de tungsteno energizados a 74 voltios con una corriente de 38.4 amperios, lo cual genera hidrógeno atómico altamente reactivo, decapando 11 fuentes de cuarzo colocadas abajo de los filamentos incandescentes a una distancia (dff) de $6 \mathrm{~mm}$, obteniendo de esta manera los precursores volátiles que se depositan y adsorben en la superficie del sustrato que se encuentra debajo de las fuentes de cuarzo a una distancia (dfs) de $8 \mathrm{~mm}[7,8,11]$. 
En el presente trabajo se utilizaron dos flujos de hidrógeno molecular $\left(\mathrm{H}_{2}\right)$ de 25 y 100 sccm para crecer dos muestras de película sencilla (P-S) etiquetadas como $\mathrm{SRO}_{25}$ y $\mathrm{SRO}_{100}$, y dos muestras de doble película (D-P) etiquetadas como $\mathrm{SRO}_{25 / 100}$ y $\mathrm{SRO}_{100 / 25}$. El tiempo de depósito $\left(\mathrm{t}_{\mathrm{d}}\right)$ para depositar una película sencilla fue de 3 minutos y el tiempo para depositar doble película fue de 5 minutos [7, 8, 11, 13, 14]. Los depósitos de SRO se llevaron a cabo sobre sustratos de silicio ( $\mathrm{Si}$ ). Posteriormente fueron tratadas térmicamente a $1100^{\circ} \mathrm{C}$ durante 60 minutos en un ambiente de $\mathrm{N}_{2}$.

El espesor y el índice de refracción de las películas SRO depositadas sobre sustratos de $\mathrm{Si}$ del tipo (V) y con (T-T) se caracterizaron con el elipsómetro Fairfield Modelo NJ 07004-2113. Por otro lado, el exceso de silicio en la composición de las películas SRO se midió con un XPS, PHI ESCA-5500 usando una fuente de radiación monocromática de $\mathrm{Al}$ con una energía de 1486 eV. Los espesores promedio, los índices de refracción y los excesos de silicio, se muestran en la Tabla 1. Mientras que los espectros FL de las películas de SRO se midieron con el espectro fluorómetro Fluoromax-3 Horiba Jobin Yvon Fluoromax-3 a temperatura ambiente en el que las muestras se excitaron utilizando una línea de excitación de $300 \mathrm{~nm}$ y la señal de emisión se recogió en el rango de 400 a $900 \mathrm{~nm}$.

\begin{tabular}{|c|c|c|c|}
\hline \multicolumn{4}{|c|}{ Del tipo (V) } \\
\hline Muestra & $\begin{array}{c}\text { Espesor } \\
\text { nm }\end{array}$ & $\begin{array}{c}\text { Índice de } \\
\text { refracción } \\
\eta\end{array}$ & $\begin{array}{c}\text { Exceso de } \\
\text { Silicio } \\
\%\end{array}$ \\
\hline $\mathrm{Si} / \mathrm{SRO}_{25}$ & 322.9 & $2.46 \pm 0.03$ & 9.9 \\
\hline $\mathrm{Si} / \mathrm{SRO}_{100}$ & 319.6 & $2.03 \pm 0.35$ & 10.6 \\
\hline $\mathrm{Si} / \mathrm{SRO}_{25 / 100}$ & 592.3 & $1.46 \pm 0.06$ & \\
\hline $\mathrm{Si} / \mathrm{SRO}_{100 / 25}$ & 578.9 & $1.51 \pm .002$ & \\
\hline \multicolumn{4}{|c|}{ Del tipo (T-T) } \\
\hline Muestra & $\begin{array}{c}\text { Espesor } \\
\text { nm }\end{array}$ & $\begin{array}{l}\text { Índice de } \\
\text { refracción }\end{array}$ & $\begin{array}{c}\text { Exceso de } \\
\text { Silicio } \\
\%\end{array}$ \\
\hline $\mathrm{Si} / \mathrm{SRO}_{25}$ & 296.3 & $1.30 \pm 0.04$ & 5.5 \\
\hline $\mathrm{Si} / \mathrm{SRO}_{100}$ & 283.5 & $1.02 \pm 0.08$ & 5 \\
\hline $\mathrm{Si} / \mathrm{SRO}_{25 / 100}$ & 583.3 & $1.43 \pm 0.31$ & \\
\hline $\mathrm{Si} / \mathrm{SRO}_{100 / 25}$ & 560.5 & $1.05 \pm 0.65$ & \\
\hline
\end{tabular}

Tabla 1 Espesores, índices de refracción y excesos de silicio de las películas SRO
Para la fabricación de la estructura MIS (Au/Si/SROP-S y D-P/ITO) como se muestran en la Figura 1. Se utilizó como contacto superior óxido de estaño dopado con indio (ITO), debido a que estas películas presentan buena transparencia y conductividad, el método de depósito utilizado fue spray pirolisis ultrasónico [2-5], mediante un nebulizador a una temperatura de depósito de $450^{\circ} \mathrm{C}$.

Mientras que para el contacto posterior se depositó Au mediante la técnica de Sputtering utilizando el equipo Denton Vacuum Desk V [26], con los parámetros de depósito de presión de vacío de 50 mTorr y una corriente DC de $25 \mathrm{~mA}$ y el blanco usado con una pureza $4 \mathrm{~N}$.

El espesor del contacto obtenido con un tiempo de depósito de $600 \mathrm{~s}$ fue de $90 \mathrm{~nm}$.

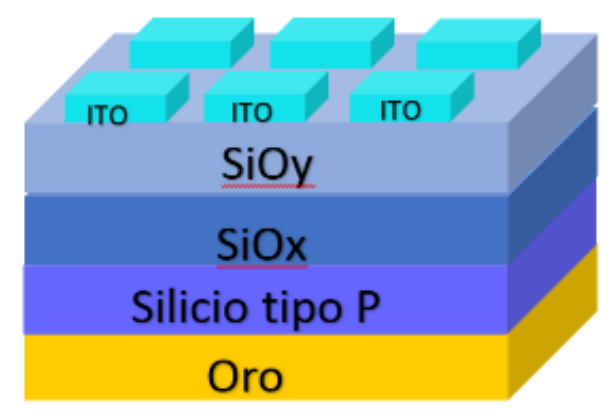

Figura 1 Estructura MIS construida con películas de SRO como capa activa depositadas por el sistema HFCVD

\section{Resultados}

La Tabla 1 exhibe una disminución en el espesor en el índice de refracción y en el exceso de silicio de las películas $\mathrm{SRO}_{\mathrm{T}-\mathrm{T}}$ con respecto al de las películas SROv, tal hecho se debe al reordenamiento a escala atómica de las películas provocado por la energía térmica generada por tratamiento térmico, así como a la desorción de las especies O-H, Si-O-Si, Si-H que están débilmente ligadas a las películas SRO y también a la formación de nanocristales de silicio (ncs-Si) y nanoclusters-Si que actúan como centros emisores radiativos, todo lo anterior es generado debido a un cambio en la estequiometría del material que lo lleva a un cambio de fase con estructura molecular similar al $\mathrm{SiO}_{2}$ [2]. 
En los espectros fotoluminiscentes (FL) observados en el Gráfico 1, es muy notorio como se incrementa la fotoluminiscencia en las películas de $\mathrm{SRO}_{\text {T-T }}$ con respecto a las películas $\mathrm{SRO}_{v}$, este incremento es más notorio en la banda roja $(750 \mathrm{~nm})$ atribuido a la existencia de confinamiento cuántico generado por los centros radiativos de baja dimensión (nanoestructuras) [6] en la estructura de las películas SRO, destacando más en las de películas sencillas que en las de doble película. Además, se observa que la película $\mathrm{SRO}_{25 / 100} \mathrm{~T}-\mathrm{T}$ aunque presenta la menor fotoluminiscencia de las películas de este tipo T-T ésta se ubica en la banda azul (450 nm), el origen de este comportamiento se atribuye a la influencia radiativa de los enlaces débiles de oxígeno (WOB), a las vacancias neutrales de oxígeno (NOV) y a algunos centros $\mathrm{B}_{2}$ $(\mathrm{SiO})_{3} \equiv \mathrm{Si} \bullet \bullet \mathrm{Si} \equiv(\mathrm{SiO})_{3},[34,5]$ inmersos en la conformación de las película $\mathrm{SRO}_{25}$ y $\mathrm{SRO}_{100}$, lo que se correlaciona con las curvas $\mathrm{I}-\mathrm{V}$ de las estructuras MIS que se muestran en el Gráfico 3.

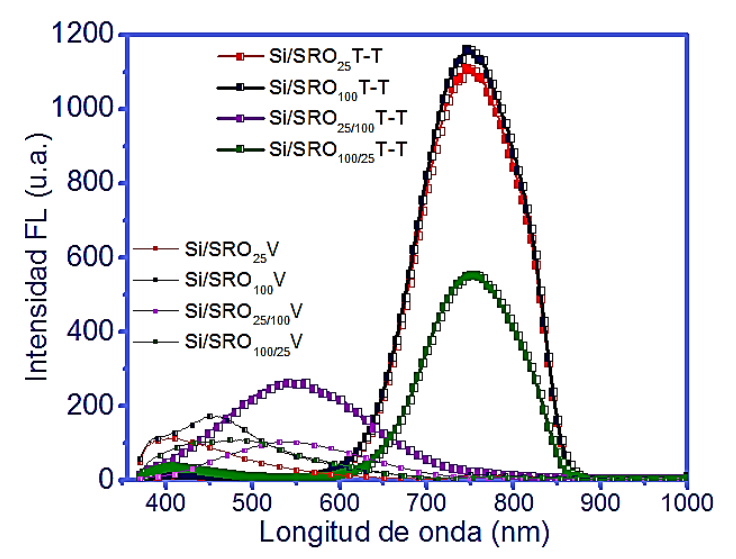

Gráfico 1 Fotoluminiscencia de películas de una capa y doble capa de $\mathrm{SRO}_{\mathrm{V} \text { y TT }}$

En el Gráfico 2 se muestran las curvas I$\mathrm{V}$ pristinas obtenidas de las estructuras MIS, todas fueron medidas con el mismo barrido de voltaje siguiendo la secuencia de $0 \mathrm{v}$ a $35 \mathrm{v}$, de $35 \mathrm{v}$ a $0 \mathrm{v}$, de $0 \mathrm{v}$ a $-35 \mathrm{v}$ y de $-35 \mathrm{v}$ a $0 \mathrm{v}$, con $100 \mathrm{~mA}$ de protección. Un aspecto relevante que se observa es que las estructuras con películas $\mathrm{SRO}_{\mathrm{V}}$ muestrean curvas disruptivas a corrientes y voltajes muy bajos, en ambas polarizaciones directa (PD) e inversa (PI), posteriormente se incrementa su corriente dependiendo del voltaje aplicado. Para las estructuras con películas SRO T-T todas las curvas I-V alcanzan mayor corriente, se muestra mayor incremento en las estructuras (a y c), coincidentemente las dos tienen la película $\mathrm{SRO}_{25}$.
Por otro lado, identificamos comportamientos diferidos contrastantes en las curvas etiquetadas con números. La curva (1) identifica el efecto de la primera medición en la cual se presenta mayor resistencia al flujo de corriente, sin embargo, en el regreso de la curva I-V se observa un estado de menor resistencia al de la curva de ida, este comportamiento se debe a la formación y anulación de caminos conductivos en el material, además, debido a que el camino de regreso no es el mismo, es ahí donde se forma una histéresis por lo que a este comportamiento se le conoce como atrapamiento de carga [8-10].

Este comportamiento se observa en todas las estructuras, pero es notado mayormente en las estructuras con películas que han sido tratadas térmicamente. La curva (2) muestra el fenómeno eléctrico identificado como bloqueo Coulómbico, el cual se caracteriza por presentar aumentos y caídas rápidas de la corriente, esto es atribuido a la existencia de electrones atrapados en estados electrónicos generados por la estructura molecular del material, este hecho genera un bloqueo en la conducción eléctrica [10-15], así como la creación y aniquilación de caminos conductivos preferenciales generados posiblemente por la estructura molecular de los Si-nps estables adyacentes y nanoclusters de silicio inestables (Si-ncls) y la posible creación de defectos debido a la ruptura de enlaces $\mathrm{Si}-\mathrm{Si}$ [9-10]. En las curvas I-V de todas las estructuras se presenta este efecto tanto en PD como en PI. La curva (3) casi siempre se observa después del Bloqueo Coulombico, se identifica como región de resistencia diferencial negativa (RDN).

La forma que presenta es una serie de brincos de corriente muy pequeños cercanos entre sí, llamado switcheo resistivo, pues según Wang, Y. et al., [10-13] esto significa que, para un rango de valores del voltaje aplicado, un aumento en el voltaje hace que la corriente disminuya en lugar de aumentar y se lleva a cabo cuando los electrones viajan a la misma velocidad de arrastre, el dominio de carga espacial ya no crece más, pero los electrones siguen su viaje y como el campo eléctrico efectivo presente en el material no es suficientemente intenso para formar dominios adicionales [10-12], entonces se forma una región de resistencia diferencial negativa, este fenómeno se observa en las estructuras que tienen las películas $\mathrm{SRO}_{\mathrm{v}}$. 
Donde la resistividad eléctrica es mayor debido a que el desorden estructural es más acentuado.

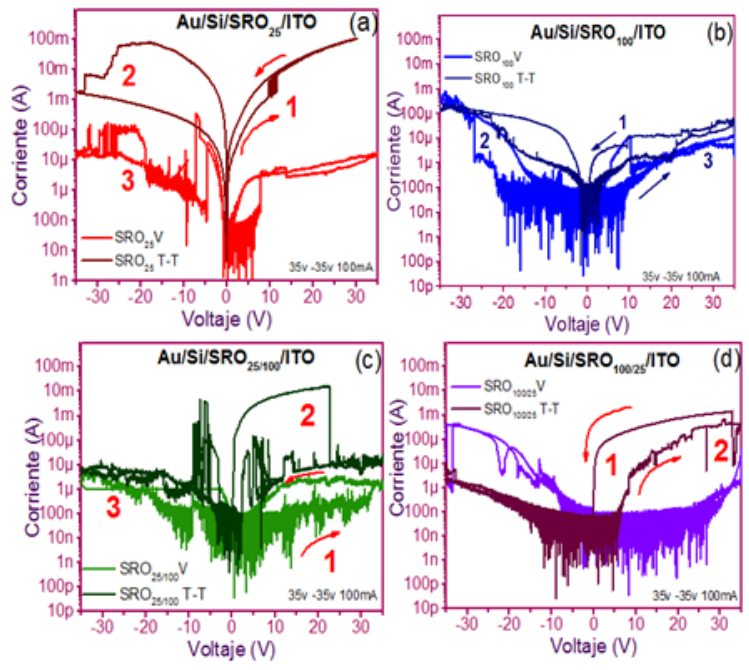

Gráfico 2 Curvas pristinas I-V de las estructuras MIS de películas sencilla y doble de $\mathrm{SRO}_{\mathrm{V} \text { y } \mathrm{TT}}$

Después de varias mediciones a temperatura ambiente de las estructuras MIS aquí estudiadas y mostradas en el Gráfico 3, estas presentan curvas típicas $\mathrm{I}-\mathrm{V}$ de las películas con ncs-Si incrustados en una matriz de $\mathrm{SiO}_{2}$ con un barrido en PI y en PD, como se corrobora en los espectros FL que se observan en la banda roja. Observamos que la corriente de las estructuras MIS del tipo (V) en PD y PI, excepto la de PI del (a), crece muy rápido con el voltaje de inicio como si fuera un corto circuito.

Sin embargo, al llegar entre los 10 y 15 volts la pendiente se reduce y el SRO ahora controla la corriente, la cual es menor que las corrientes que alcanzan las estructuras MIS con T-T. Estas estructuras MIS con T-T muestran también incrementos de corriente a bajos voltajes hasta alcanzar el régimen de alta conducción como es esperado en estos dispositivos [9-13], lo que indicaría que el tener altas corrientes desde el inicio se debe al tratamiento térmico proporcionado a las películas SRO.

Algunos autores [8-10] atribuyen el régimen de alta conducción al transporte de carga en las películas SRO, que contienen Si-ncs y forman caminos de conducción.
Además, señalan que la carga y descarga de los capacitores nanocristalinos equivalentes tienen un gran impacto en los estados de encendido y apagado de los estados de conducción, estos efectos lo han relacionado con el efecto de bloqueo Coulombico (CB) [12,13], mismo que se observa muy poco en estas curvas $\mathrm{I}-\mathrm{V}$ con $\mathrm{T}-\mathrm{T}$ debido a que los caminos conductivos fueron formados. No obstante, existen dos mecanismos que pueden producir estos cambios en los estados de conducción ambos relacionados a la carga y descarga de los nanocristales los cuales son responsables de las oscilaciones y saltos abruptos en las curvas de corriente contra voltaje. Uno de ellos debido a la formación de un "efecto de campo" en los caminos de conducción y el otro debido a efectos capacitivos en ellos [10-15].
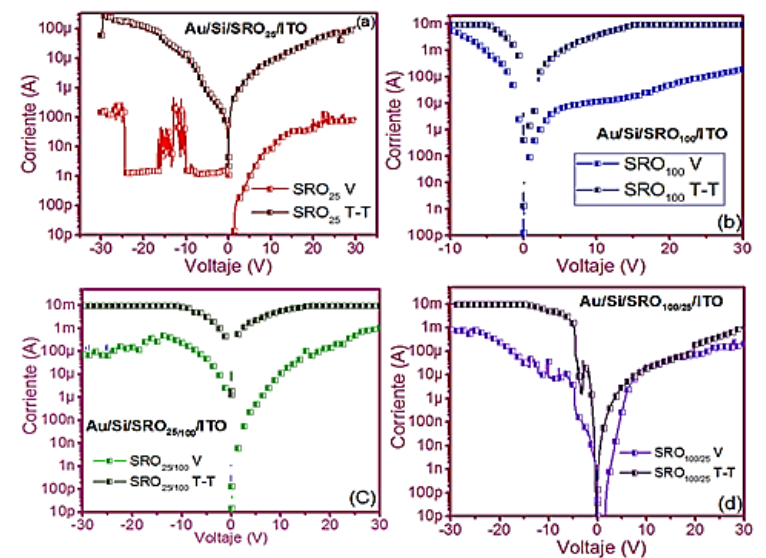

Gráfico 3 Curvas I-V de las estructuras MIS de película sencilla y doble de $\mathrm{SRO}_{\mathrm{v} \text { y } \mathrm{TT}}$

\section{Conclusiones}

El análisis electro-óptico de las estructuras MIS muestra que están conformadas por películas nanométricas de SRO-HFCVD sencillas y dobles sin y con tratamiento térmico, las cuales muestran efectos luminiscentes en regiones del espectro que son de interés tecnológico.

Con estos resultados podemos inferir que el sistema HFCVD utilizado para obtener películas de SRO con excelentes propiedades ópticas y estructurales resulta ser una relevante alternativa. Las estructuras de doble película de SRO mejoran la respuesta fotoluminiscente en comparación con las de película sencilla. 
Así mismo, las propiedades eléctricas de las estructuras cuantificadas a través de la conductividad eléctrica mejoran cuando las películas con alto contenido de exceso de silicio fueron intercaladas como películas emisoras, generando estructuras MIS con curvas I-V características, en las cuales se han observado fenómenos eléctricos peculiares debido a la compleja estructura molecular de las películas-

SRO los cuales no están presentes en materiales dieléctricos homogéneos ordinarios, y estos son: anulación de caminos conductivos en el material, brecha de bloqueo Coulómbico y resistencia diferencial negativa (RDN).

\section{Reconocimientos}

Este trabajo ha sido parcialmente soportado por CONACyT-CB-255062, PROFOCIE 2019 y VIEP-LULJ-EXC-2019. Los autores agradecen a CIDS

\section{Referencias}

[1] Benítez-Lara, A. et al., (2015). Silicon rich oxide powders by HWCVD: Its optical and morphological properties. Adv. Powder Technol. 26, 163-168.

[2] H. P. Martínez-Hernández, et al., (2020). Spectroscopic and Microscopic Correlation of SRO-HFCVD Films on Quartz and Silicon. Crystals 2020, 10, 127; 19.

[3] Valerdi, D.E.V.; et al., (2014). Twofold SiOx Films deposited by HFCVD: It is Optical, Compositional and Electrical Properties. Procedia Eng., 87, 168-171.

[4] DiMaria, et al., (1983). Charge transport and trapping phenomena in $\mathrm{o}_{-}$-stoichiometric silicon dioxide films. J. Appl. Phys. 1983, 54, 5801-5827.

[5] Canham, L. T. (1990). Silicon quantum wire array fabrication by electrochemical and chemical dissolution of wafers. Appl. Phys. Lett. 57, 1046

[6] E. Quiroga, et al., (2009). Structural characteristics of a multilayer of silicon rich oxide (SRO) with high Si content prepared by LPCVD, Phys. Status Solidi A, 206, $\mathrm{n}^{\circ} 2$, 263-269.
[7] Chang, Y. F., et al., (2014). Intrinsic SiOxbased unipolar resistive switching memory. I. Oxide stoichiometry effects on reversible switching and program window optimization. Journal of Applied Physics, 116(4), 043708.

[8] Wang, Y., et al., (2013). Resistive switching mechanism in silicon highly rich $\mathrm{SiOx}(\mathrm{x}<$ 0.75 ) films based on silicon dangling bonds percolation model. Applied Physics Letters, 102(4), 042103.

[9] Zhou, F., et al., (2015). Stabilization of multiple resistance levels by current-sweep in SiOx-based resistive switching memory. Applied Physics Letters, 106(6), 063508.

[10] Wang, Y., et al., (2014). The $\mathrm{x}$ dependent two kinds of resistive switching behaviors in SiOx films with different $\mathrm{x}$ component. Applied Physics Letters, 104(1), 012112.

[11] Fu-Chien Chiu (2014) A Review on Conduction Mechanisms in Dielectric Films

[12] J. Juvert, et al., (2009) Electroluminiscencia bajo tensión continua de dispositivos MOS basados en nanocrsitales de silicio obtenidos por LPCVD.

[13] J. Alberto Luna López, et al., (2003) "Efecto de la Carga Atrapada obtenida mediante Inducción de Alto Voltaje en la Fotocorriente del Dispositivo Al/SRO/Si", publicado en las Memorias del Congreso de Ingeniería Eléctrica CIE2003. Pag.177-180, CINVESTAV, 3 al 5 de septiembre del 2003.

[14] Zhenrui Yu, et al., (2003), Charge trapping phenomenon in $\mathrm{Al} / \mathrm{SRO} / \mathrm{Al}$ on $\mathrm{Si}$ structure by lateral electrical stress", Superficies y Vació, Vol 16(4), pp.25-29.

[15] F. H Hielsher et al., (1969). "Non equilibrium $\mathrm{C}-\mathrm{V}$ and I-V Characteristics of Metal-Insulator-Semiconductor Capacitors, Solid State Electronics, Vol. 22, pp. 527538. 